\title{
Accuracy and Resolution of ALOS Interferometry: Vector Deformation Maps of the Father's Day Intrusion at Kilauea
}

\author{
David T. Sandwell, David Myer, Robert Mellors, Masanobu Shimada, Senior Member, IEEE, \\ Benjamin Brooks, and James Foster
}

\begin{abstract}
We assess the spatial resolution and phase noise of interferograms made from L-band Advanced Land Observing Satellite (ALOS) synthetic-aperture-radar (SAR) data and compare these results with corresponding $\mathrm{C}$-band measurements from European Space Agency Remote Sensing Satellite (ERS). Based on cross-spectral analysis of phase gradients, we find that the spatial resolution of ALOS interferograms is $1.3 \times$ better than ERS interferograms. The phase noise of ALOS (i.e., line-of-sight precision in the $100-5000-m$ wavelength band) is $1.6 \times$ worse than ERS (3.3 mm versus $2.1 \mathrm{~mm})$. In both cases, the largest source of error is tropospheric phase delay. Vector deformation maps associated with the June 17, 2007 (Father's day) intrusion along the east rift zone of the Kilauea Volcano were recovered using just four ALOS SAR images from two look directions. Comparisons with deformation vectors from 19 continuous GPS sites show rms line-of-site precision of $14 \mathrm{~mm}$ and rms azimuth precision (flight direction) of $71 \mathrm{~mm}$. This azimuth precision is at least $4 \times$ better than the corresponding measurements made at $\mathrm{C}$-band. Phase coherence is high even in heavily vegetated areas in agreement with previous results. This improved coherence combined with similar or better accuracy and resolution suggests that L-band ALOS will outperform C-band ERS in the recovery of slow crustal deformation.
\end{abstract}

Index Terms-Crustal deformation, phase noise, radar interferometry, synthetic aperture radar (SAR).

\section{INTRODUCTION}

$\mathbf{P}$ ALSAR is the first L-band synthetic aperture radar (SAR) having the duration and orbital accuracy needed to monitor slow crustal deformation globally [1], [2]. The main advantages of the L-band (236-mm wavelength) PALSAR over C-band (56-mm wavelength) are as follows: deeper penetration of vegetated areas results in less temporal decorrelation, enabling

Manuscript received November 10, 2007. Current version published October 30, 2008. This work was supported by the Southern California Earthquake Center and the National Science Foundation under Grant EAR 0841772.

D. T. Sandwell and D. Myer are with the Scripps Institution of Oceanography, University of California, San Diego, La Jolla, CA 92093-0225 USA (e-mail: dsandwell@ucsd.edu).

R. Mellors is with the Department of Geological Sciences, San Diego State University, San Diego, CA 92182-1020 USA.

M. Shimada is with the Advanced Land Observing Satellite (ALOS) Science Project, JAXA/EORC, Tsukuba 305-8505, Japan.

B. Brooks and J. Foster are with the Hawaii Institute of Geophysics, University of Hawaii, Honolulu, HI 96822 USA.

Color versions of one or more of the figures in this paper are available online at http://ieeexplore.iee.org.

Digital Object Identifier 10.1109/TGRS.2008.2000634
TABLE I

COMPARISON OF CRITICAL BASELINE

\begin{tabular}{|l|c|c|c|}
\hline look angle & $23^{\circ}$ & $34^{\circ}$ & $41^{\circ}$ \\
\hline $\begin{array}{l}\text { ERS/ENVISAT } \\
16 \mathrm{MHz}\end{array}$ & $1.1 \mathrm{~km}$ & 2.0 & 2.9 \\
\hline $\begin{array}{l}\text { ALOS FBD } \\
14 \mathrm{MHz}\end{array}$ & 3.6 & 6.5 & 9.6 \\
\hline $\begin{array}{l}\text { ALOS FBS } \\
28 \mathrm{MHz}\end{array}$ & 7.3 & 13.1 & 18.6 \\
\hline
\end{tabular}

ERS/ENVISAT - altitude $=790 \mathrm{~km}$, wavelength $=56 \mathrm{~mm}$

ALOS - altitude $=700 \mathrm{~km}$, wavelength $=236 \mathrm{~mm}$

Shaded area is most common mode for interferometry.

interferograms to have longer time separation [3]; and the longer wavelength increases the critical baseline, resulting in more usable interferometric pairs. The potential disadvantages are as follows: the lower fringe rate may result in less precise crustal-motion measurements; and the ionospheric refraction should be $16.5 \times$ worse at L-band versus C-band. The path delays caused by water vapor in the troposphere are independent of wavelength, so this distortion will affect both systems equally [4].

In addition to these fundamental wavelength-dependent issues, PALSAR is operated in a number of different modes that could both enhance and detract from its interferometric capabilities [1]. In particular, the fine-beam single polarization (FBS- $\mathrm{HH}, 28-\mathrm{MHz}$ bandwidth) has $2 \times$ better range resolution than most previous InSAR instruments, which further increases the critical baseline and could improve the spatial resolution of the interferograms. The fine-beam dual polarization (FBD- $\mathrm{HH}$ and $\mathrm{HV}, 14 \mathrm{MHz}$ ) has $2 \times$ worse range resolution than the FBS mode. Table I shows the critical baseline, beyond which phase coherence drops to zero. The orbits of ERS and ENVISAT are controlled within about a 1-km-diameter tube, so not all pairs of SAR images can be used for interferometry, because they commonly have baselines greater than half the critical value [5]. Initially Advanced Land Observing Satellite (ALOS) was controlled within about a 3-km tube, but since early 2007 , the tube diameter has reduced to $1 \mathrm{~km}$. Another important issue related to interferometry is adequate control of the squint angle of the spacecraft to achieve a large overlap in the Doppler spectrum. For the nine repeat passes discussed as follows, the maximum variation in the Doppler away from the mean value is only $3 \%$ of the pulse repetition frequency (PRF). Because of this accurate control of the spacecraft attitude 
combined with baselines that are always less half the critical, every SAR pair can be used for interferometry.

In this paper, we use ALOS PALSAR data from the first 1.5 years of the mission to evaluate the following three quality parameters of the interferograms: the spatial resolution of the phase, the short wavelength "instrument" phase noise, and the overall geodetic accuracy of the system, including the effects of orbital and other errors. This analysis of the resolution and noise of the phase derived from an interferogram is very different from the more traditional analyses of the resolution and noise of the backscatter derived from a single SAR image [6]. In the backscatter-resolution analysis, one is interested in the ability to resolve two closely spaced reflectors. For this phase-resolution analysis, we are interested in the ability to resolve two closely spaced topographic or deformation features.

The spatial resolution of the phase is estimated by crossspectral analysis of independent interferograms having baselines of several hundred meters; this requires at least four repeated SAR images. Our approach follows the standard coherence method [7], [8] available in MATLAB as the function mscohere(). The phase due to the topography of the Earth serves as a signal, which is common to both interferograms. The properly scaled difference of the two interferograms is a measure of the noise. The analysis of signal-to-noise ratio (SNR) versus spatial wavelength provides an estimate of resolution in both range and azimuth directions. An identical analysis was performed using ERS interferometry [9], enabling a direct comparison of the resolution capabilities of ERS and ALOS. The wavelength where the coherence falls to 0.2 is defined as the resolution [8], and this also determines the initial low-pass (multilook) filter to be applied to all interferograms. After the low-pass filter is designed, the remaining phase noise is estimated from the difference interferograms. To isolate the radar "instrument" noise from the atmospheric and orbital phase variations, we high-pass filter the differentiated interferograms for wavelengths shorter than $5 \mathrm{~km}$ and scale the phase by the appropriate wavelength to form line-of-sight (LOS) difference maps. Finally, we assess the overall accuracy of the ALOS InSAR system by constructing a vector deformation map associated with the June 17, 2007 (Father's day) dike-injection event at the Kilauea Volcano, Hawaii. Within the interferograms, 19 continuous GPS stations provide ground-truth deformation measurements. For all these analyses, interferograms were constructed from images in the high-bandwidth (FBS), lowbandwidth (FBD), and mixed modes.

\section{Data AnAlysis}

\section{A. Unique Capabilities of PALSAR}

The unique capabilities of ALOS PALSAR require some refinement of the commonly used InSAR processing methods. 1) Proper focus of the imagery, particularly at the higher FBS bandwidth, requires an accurate description of the range migration of a target while it is in the synthetic aperture. In our simple range Doppler processor, this corresponds to an improved estimate of the Doppler rate parameter (details are provided in Appendix A). 2) As shown in Table I, ALOS interferometry can tolerate long perpendicular baselines; however, this produces an elevation-dependent range shift that must be corrected to retain interferometric coherence (details are provided in Appendix B). 3) The acquisition plan for PALSAR has an alternation between the low- (FBD) and high-bandwidth (FBS) acquisition modes. In order to make FBD2FBS interferograms, we have developed a simple $2 \mathrm{X}$ interpolation algorithm to convert the raw waveform data between FBD $(\mathrm{HH}, 14 \mathrm{MHz})$ and FBS $(\mathrm{HH}, 28-\mathrm{MHz}$ bandwidth) modes. The FBD-to-FBS conversion approach is to Fourier transform each complex radar echo and double the length of the array in the frequency domain. The values of the added high frequencies are set to the complex number zero. The zero-padded data are inverse Fourier transformed, resulting in a new radar echo that matches the length and sampling rate of the FBS data. This algorithm provides a smooth interpolation yet retains the complex values of the original FBD data at every other data point. One could also resample the singlelook complex image that is the output of the SAR processor to achieve the same result.

The motivation for this resolution and accuracy analysis is to understand the strengths and limitations of ALOS interferometry and also to optimize InSAR processing methods. In particular, the estimates of spatial resolution and noise are used to design a prestack low-pass filter that is applied to the real and imaginary parts of the interferogram prior to computing phase and coherence. We have not yet assessed the longer wavelength $(>5 \mathrm{~km})$ error characteristics in the ALOS interferograms. Since we expect that the atmospheric noise at L-band will be the same as C-band, this issue will not be addressed in this paper. As discussed earlier, we expect a significant new (not usually evident at C-band) error contribution from the ionosphere [10], [11]. In addition, there will be long-wavelength contribution from orbit error. Separation of the ionospheric and orbital error will require the analysis of many more interferograms in concert with phase-error estimates derived from total electron concentration of the ionosphere [12]. All of these results will be helpful in optimizing future interferometric satellites such as DESDynI for the recovery of crustal-deformation signals.

\section{B. Resolution and Noise, California}

To assess the resolution and noise characteristics of ALOS interferometry, we have selected an area in southern California that has been the site of several other InSAR resolution and noise studies (Fig. 1). This area also contains permanent radar corner reflectors at Pinyon Flat observatory (star in Fig. 1) that are used for radiometric and geometric calibration of PALSAR. Unlike most areas of the Earth where there are not yet more than two to three repeat images, JAXA has imaged the Pinyon area on all over flights ( $\sim 41 \times$ in 1.5 years). PALSAR data have been collected $9 \times$ along an ascending track (T213), which contains sections of the San Jacinto, San Andreas, and Pinto Mountain faults (blue box in Fig. 1). This area has more than $3000 \mathrm{~m}$ of relief and includes forested and desert areas; images were acquired during both dry and snow-covered conditions. The area slightly to the east (green box in Fig. 1) has been imaged $74 \times$ at C-band by ERS-1/2. These L- and C-band data are optimal for exploring the strengths and limitations of L- and C-band 


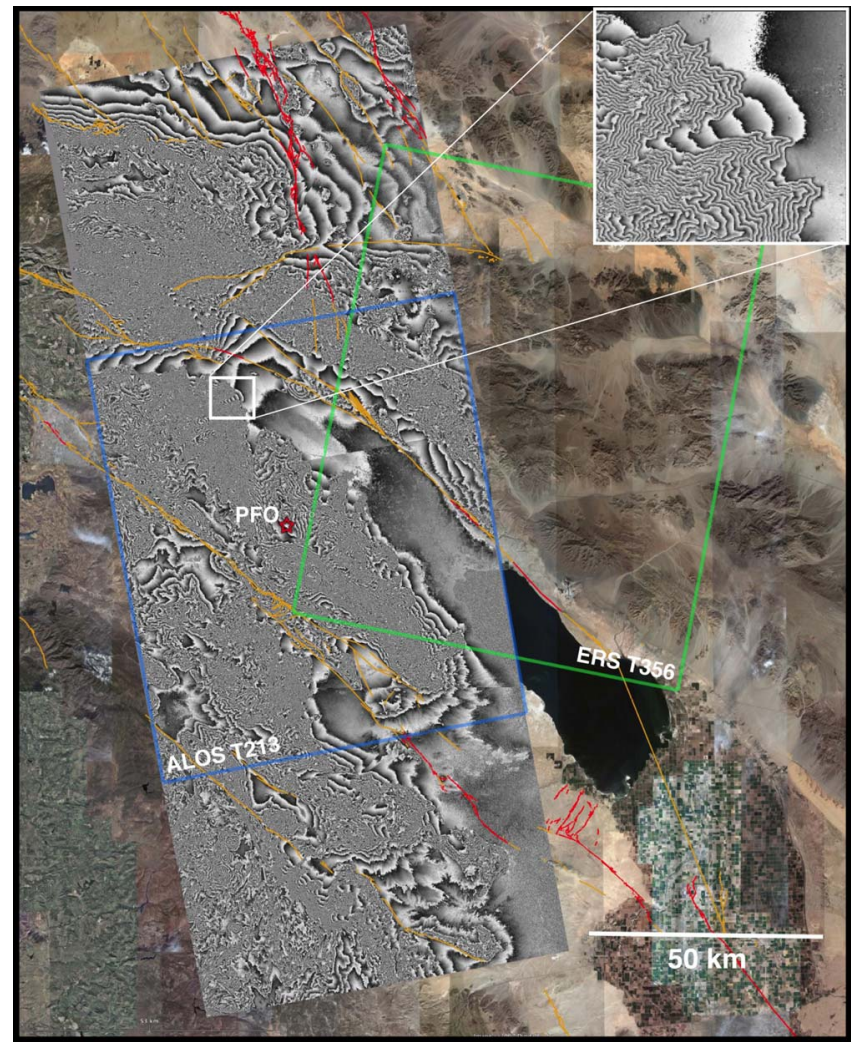

Fig. 1. Example interferogram along ALOS track 213, frames 0650-0670, and look angle $34.3^{\circ}$. The location of the Pinyon Flat radar corner reflector is shown as a red star. The blue box marks the boundaries of T213 F0660. A nearby ERS scene (T356 F2907) is marked by the green box. Red lines mark currently active faults, and the yellow lines are active faults that have not ruptured in historical times. The inset box shows the high fringe rate associated with the $3000 \mathrm{~m}$ of relief in the area. One fringe corresponds to $86 \mathrm{~m}$ of elevation change.

interferometry. It should be noted that this analysis is limited to this one area where enough repeat passes are available. Resolution and accuracy results may be different for other areas (e.g., urban or vegetated) where surface reflective properties are different. In addition, we do not sample the complete range of look angle, temporal baseline, or perpendicular baseline.

A plot of perpendicular baseline versus time for these nine acquisitions is shown in Fig. 2. All images were aligned to the master 5575 FBS so that any interferometric combination could be constructed. The mean Doppler centroid (values given in Fig. 2) of the images is $103 \mathrm{~Hz}$, which is a small fraction of the PRF of $2155 \mathrm{~Hz}$. The maximum difference from the mean is only $66 \mathrm{~Hz}$, so there is nearly complete overlap of the Doppler spectra. The resolution analysis requires moderate baselines, so there will be a common topographic signal. These pairs are connected by blue lines. The 3562_FBS to 4233_FBS pair labeled (0) serves as the reference topographic phase to be added and subtracted from the other pairs for the resolution analysis. Note that the other pairs form all possible mode combinations: 1) 4904_FBS to 5575_FBS, 2) 5575_FBS to 7588_FBD, and 3) 7588_FBD to 8259_FBD. The noise analysis was performed using the two pairs having shorter perpendicular baselines 4233_FBS to 6246_FBS and 6246_FBS to 8259_FBD. In addition to these interferograms used in the resolution and noise analyses, we formed many other interferograms and found gen-

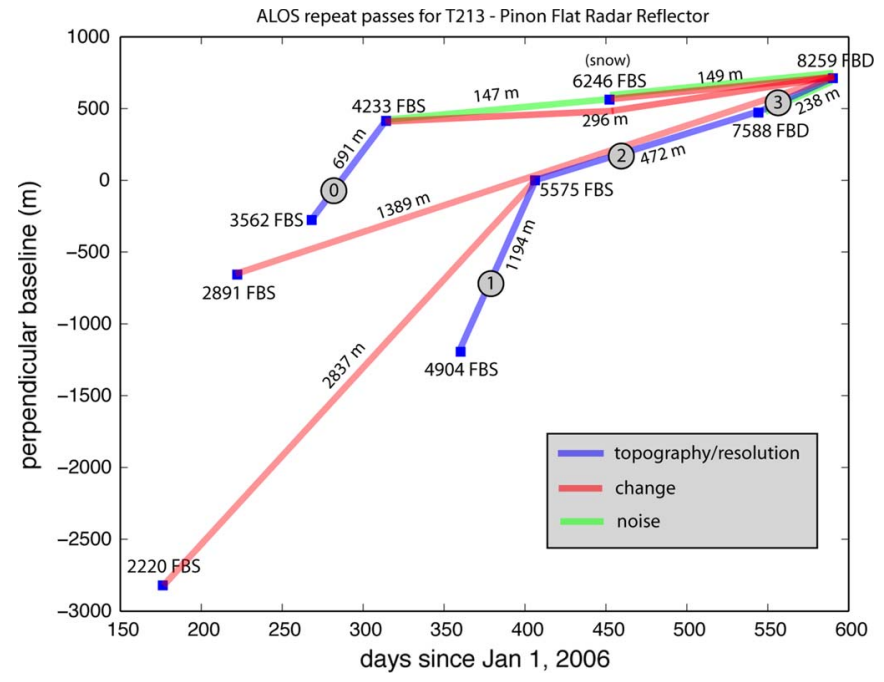

Fig. 2. Perpendicular baseline versus time. All images were aligned with the master scene from orbit 5575. The first seven acquisitions are in the highbandwidth (FBS) mode, while the last two are the low-bandwidth mode (FBD). Pairs connected by blue lines have significant topographic phase and were used for estimating spatial resolution. Pairs connected by green lines were used to assess short wavelength noise. Pairs connected by red lines are suitable for change detection. The PRF of all the images is between 2155 and $2160 \mathrm{~Hz}$. The Doppler centroid for each of the nine images is $169,96,99,93,85,95,105$, 96, and $102 \mathrm{~Hz}$. All images were processed with a common Doppler centroid of $100 \mathrm{~Hz}$.

erally excellent coherence in all cases. As discussed as follows, the quality of the long-baseline interferograms is limited by the errors in the topographic phase.

The resolution analysis was performed in exactly the same way as a previous study using ERS repeat data, so the results could be directly compared [9]. The standard approach to adding or subtracting wrapped phase requires phase unwrapping, scaling the phase by the ratio of the perpendicular baseline, and finally, forming the average [13]. Unique phase unwrapping is not always possible, because areas of the interferogram may not be coherent due to high relief or wavelength-scale surface changes between the two observation times [14]. In this paper, we avoid phase unwrapping by computing the phase gradient. Using the chain rule, the gradient of the phase $\phi=\tan ^{-1}(I / R)$ is [15]

$$
\nabla \phi(\mathbf{x})=\frac{R \nabla I-I \nabla R}{R^{2}+I^{2}}
$$

where $R(\mathbf{x})$ and $I(\mathbf{x})$ are the real and imaginary components of the interferogram. Unlike the wrapped phase, which contains many $2 \pi$ jumps, the real and imaginary components of the interferogram are usually continuous functions, and thus, the gradient can be computed with a convolution operation and a well-designed derivative operator; a first difference derivative operator is inadequate because of the large sidelobes in its spectrum [9].

Our objective is to determine the resolution of both the ERS and ALOS interferograms and use this to design a low-pass filter that will suppress noise but retain the signal at high spatial wavenumber that may become available after stacking many interferograms. The repeat-track analysis method [7], [8], [16] was used to evaluate the signal and noise characteristics of the 


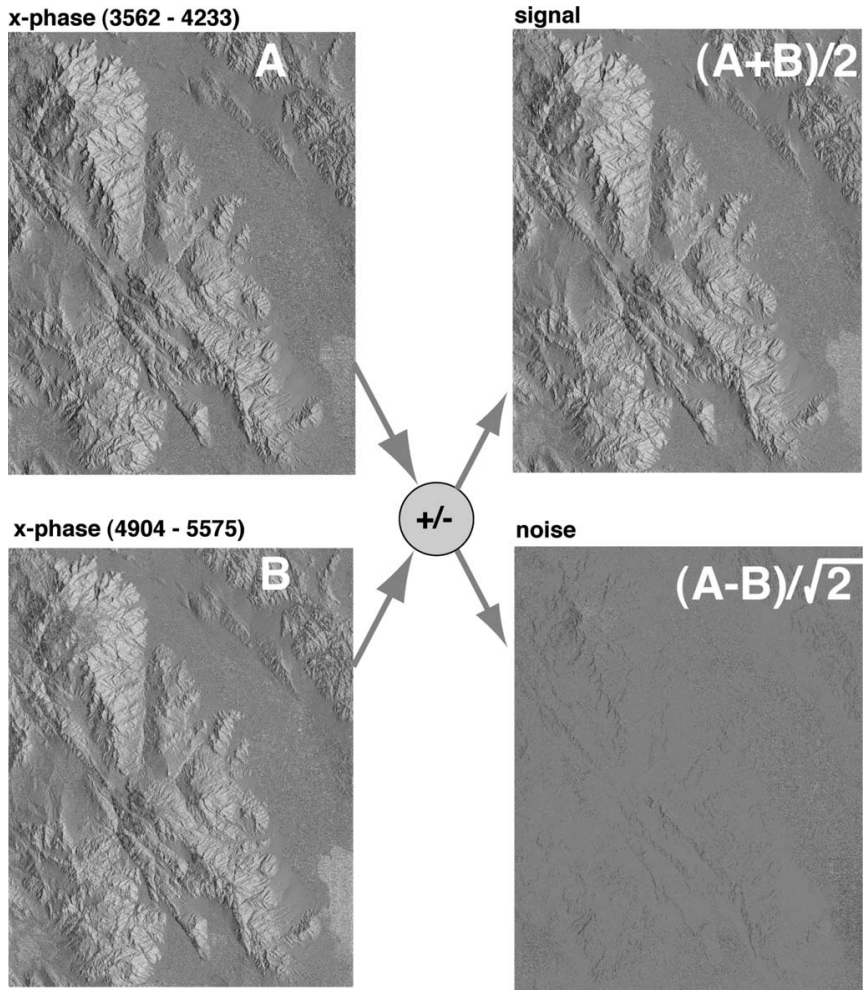

Fig. 3. Range component of phase gradient for two independent ALOS interferograms. These were scaled to $100-\mathrm{m}$ baseline. White and black correspond to $\pm 0.3 \mathrm{rad}$ per pixel.

phase-gradient data as a function of spatial wavenumber. For the analysis, we selected two interferograms generated from four independent SAR images. An example of the $x$-component of the phase gradient for two independent ALOS interferograms is shown in Fig. 3. Consider the range coherence first; the $x$ components of the phase gradient along corresponding rows (length 2048) of the two interferograms are loaded into vectors $s_{1}$ and $s_{2}$, where $s_{2}$ is scaled by the ratio of the perpendicular baselines. If there is no noise, the data vectors should be equal to their common signal $S$, but because of many factors, each vector has a noise component $n_{1}$ and $n_{2}$. The model is [8]

$$
s_{1}=S+n_{1} \quad \mathrm{~s}_{2}=S+n_{2} .
$$

An estimate of the signal is the average of the two $x$-phase segments $S=\left(s_{1}-s_{2}\right) / 2$, while an estimate of the noise is the difference between two $x$-phase segments $d=\left(n_{1}-n_{2}\right) / \sqrt{2}$. Each segment of $x$-phase data plus their sums and differences were Hanning windowed and Fourier transformed [7], [16]. Spectral estimates from 500 independent rows/columns, from regions of visual good correlation, were ensemble averaged to form smooth power spectra, cross spectra, and coherence segments; only every tenth row was analyzed to ensure that the profiles are statistically independent. The range ( $x$-phase) and azimuth ( $y$-phase) gradient data were treated separately. The results for ERS [9] are shown in Fig. 4, where the signal power, noise power, and coherence are plotted versus spatial wavenumber. Note that, to obtain the power in the phase rather than the phase gradient, one should divide each curve by the wavenumber squared. The derivative operation has no effect on the esti-
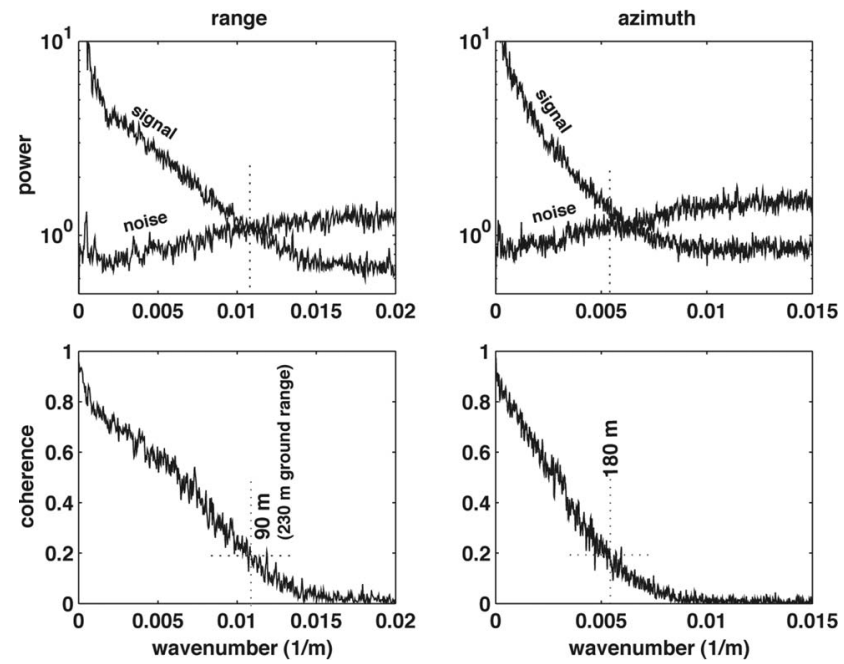

Fig. 4. Cross spectral analysis between phase gradients from ERS-1/2 tandem interferograms reveals the signal and noise as a function of wavenumber (upper) as well as the coherence versus wavenumber (lower). These ERS tandem interferograms have one-day time separation and similar perpendicular baselines of 98 and $125 \mathrm{~m}$. For uncorrelated noise, a coherence of 0.2 marks an SNR of one and provides a good estimate of the wavelength resolution of the data. ERS ground-range resolution is $230 \mathrm{~m}$, while azimuth resolution is $180 \mathrm{~m}$. Stacking may provide better resolution, so we design filters to cut wavelength shorter than $100 \mathrm{~m}$ from the full-resolution interferogram.

mates of coherence, and it provides a natural means of "prewhitening" prior to Fourier analysis.

The signal power (Fig. 4, upper plots) decreases rapidly with increasing wavenumber in both range and azimuth, reflecting the power spectra of the common topographic signal. The noise spectra increase with increasing wavenumber between 0 and $0.01 \mathrm{~m}^{-1}$ (100-m wavelength) reflecting the "whitening" provided by the derivative operation. At wavenumbers greater than $0.01 \mathrm{~m}^{-1}$, the noise spectra begin to flatten, reflecting the 66-m Gaussian prefilter [9]. The coherence (Fig. 4, lower plots) reflects the SNR and provides an estimate of the resolution of the data in both range and azimuth. In slant range, the coherence falls below 0.2 at a wavelength of $90 \mathrm{~m}(\sim 230 \mathrm{~m}$ in ground range), while in azimuth, the coherence falls below 0.2 at a wavelength of $180 \mathrm{~m}$.

The corresponding analysis for ALOS data is shown in Fig. 5, and the combined results are summarized in Table II. In general, ALOS has better spatial resolution than ERS, but there is an important factor that affects the ALOS results. The repeat interferograms have widely differing perpendicular baseline $(1194,472$, and $238 \mathrm{~m})$. Since none of these baselines approach the critical baseline for $34.3^{\circ}$ look angle (Table I), the amplitude in the signal will increase linearly with increasing baseline, while the noise may remain relatively constant. Therefore, the better resolution of the FBS2FBS interferogram with respect to the other two mode combinations may simply be a consequence of the longer baseline. As we will see next, the noise floor of ALOS in millimeters is slightly higher than the noise floor in ERS, so the improvement in resolution of ALOS with respect to ERS is due to the longer baseline (higher signal) available for ALOS. Nevertheless, the overall results show that the resolution capabilities of ALOS are somewhat better than ERS. Given these resolution estimates, we have adopted a standard low-pass filter for both ALOS and ERS interferometry that is a Gaussian 

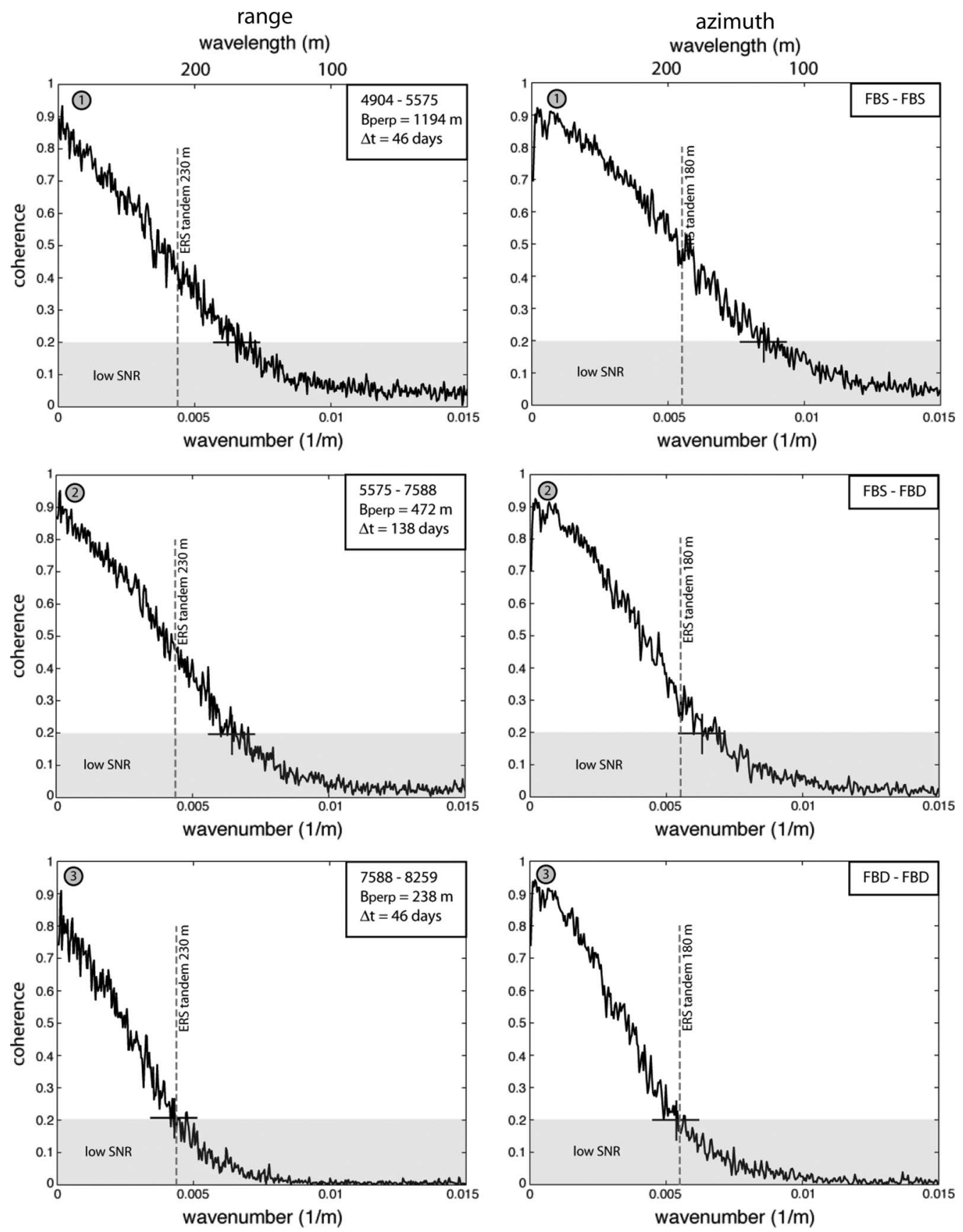

Fig. 5. Cross spectral analysis between phase gradients from ALOS interferograms reveals the coherence versus wavenumber for range and azimuth directions and also for each of the three possible mode combinations. For uncorrelated noise, a coherence of 0.2 marks an SNR of one and provides a good estimate of the wavelength resolution of the data. ALOS ground-range resolution is generally better than ERS resolution. Stacking may further improve resolution, because the noise level will be reduced, so we design filters to cut wavelength shorter than $100 \mathrm{~m}$ from the full-resolution interferogram.

function that has a 0.5 gain at a wavelength of $100 \mathrm{~m}$ in both range and azimuth. As the temporal baseline increases, the noise level may increase, which may necessitate longer filters to isolate the part of the interferogram having SNR $>1$. Note that the initial filter with a 100-m cutoff wavelength limits the ultimate pixel resolution of our interferograms to about $25 \mathrm{~m}$, and we really only trust features having length scale greater than $50 \mathrm{~m}$.

As discussed in the introduction, a potential disadvantage of L-band with respect to C-band interferometry is that the phase signal per unit of deformation is $4 \times$ lower. If $\mathrm{L}$ - and
C-band have the same phase noise, then L-band interferometry will be $4 \times$ worse at recovering small deformations such as those associated with fault creep or triggered slip. To estimate the actual loss of range precision at L-band, we formed three ALOS interferograms and compared them with five ERS interferograms of almost the same area containing the Salton Sea, Coachella Valley, dry desert areas, and the high mountains (Fig. 6). Both sets of interferograms were converted to LOS deformation using the appropriate radar wavelength. The interferograms were low-pass filtered at $100-\mathrm{m}$ spatial wavelength as discussed earlier. In both cases, the main contribution to 
TABLE II

RESOLUTION ALOS VERSUS ERS

\begin{tabular}{|l|c|c|c|c|c|}
\hline \multicolumn{1}{|c|}{ interferogram } & mode & $\begin{array}{c}B_{\perp} \\
(\mathrm{m})\end{array}$ & $\begin{array}{c}\Delta t \\
(\text { days })\end{array}$ & $\begin{array}{l}\text { resolution } \\
\text { range (m) }\end{array}$ & $\begin{array}{l}\text { resolution } \\
\text { azimuth } \\
(\mathrm{m})\end{array}$ \\
\hline A_3562-A_4233 & FBS2FBS & 691 & 46 & - & - \\
\hline A_4904-A_5575 & FBS2FBS & 1194 & 46 & 151 & 116 \\
\hline A_5575-A_7588 & FBS2FBD & 472 & 138 & 156 & 158 \\
\hline A_7588-A_8259 & FBD2FBD & 238 & 46 & 238 & 181 \\
\hline $\begin{array}{l}\text { E2_03259- } \\
\text { E1_22932 }\end{array}$ & & 98 & 1 & - & - \\
\hline $\begin{array}{l}\text { E2_02257- } \\
\text { E1_21930 }\end{array}$ & & 126 & 1 & 230 & 180 \\
\hline
\end{tabular}

ERS analyses from [9]

the phase error was atmospheric phase delay, which can be typically $10-\mathrm{mm}$ rms. In addition, there is a somewhat smaller but important contribution to the phase error due to errors in the topographic phase. From the geometry of a repeat-pass SAR above a spherical surface, one can derive an equation relating the error in the LOS $l$ to the error in the topography $h$

$$
\frac{\partial l}{\partial h}=\frac{R_{e} B_{\perp}}{\rho b \sin \theta}
$$

where $R_{e}$ is the local Earth radius, $B_{\perp}$ is the perpendicular baseline, $\rho$ is the range, $b$ is the spacecraft orbital radius, and $\theta$ is the look angle. Note that the scaling between topography and LOS is independent of radar wavelength. In the case of ERS with a $100-\mathrm{m}$ perpendicular baseline and $23^{\circ}$ look angle, the scale factor is $2.8 \times 10^{-4}$. Therefore, a 10 -m error in topography will map into a $2.8-\mathrm{mm}$ error in LOS. In the case of ALOS with a $100-\mathrm{m}$ baseline and $34^{\circ}$ look angle, the scale factor is nearly the same $1.9 \times 10^{-4}$. Our ALOS interferograms, typically, have $150-\mathrm{m}$ baseline, so the $10 \mathrm{~m}$ of topography error will map into the same 2.8-mm error in LOS. The conclusion is that, while ALOS has a much larger critical baseline than ERS, the need for highly accurate topography or shorter baselines is the same in both cases.

To minimize the phase errors due to atmosphere and topography, we high-pass filtered both sets of interferograms using a Gaussian filter with a 0.5 gain at a $5000-\mathrm{m}$ wavelength. These residual interferograms are shown in Fig. 6. In areas of high topography, the topographic phase error still dominates even at these small scales. Topographic phase error is less of a problem for ERS, because we used very high accuracy topographic phase, which has constructed the radar coordinates by stacking the residual phase from 25 interferograms [17]. For ALOS, the topographic phase was constructed from SRTM topography data at 30-m postings [18], which is known to have errors of $7 \mathrm{~m}$, averaged over North America and perhaps much larger areas in rugged terrain. We also tried using the National Elevation Data, which is available at $10-\mathrm{m}$ postings in the U.S., but the results (rms residual) were worse than using the SRTM data. It may be possible that the "errors" in the elevation models are due to real differences in the elevation of the topographic surfaces recovered at C- (SRTM) and L-band. Unfortunately, there are not enough ALOS interferograms to stack to construct an accurate topographic phase. We do not completely understand this issue.

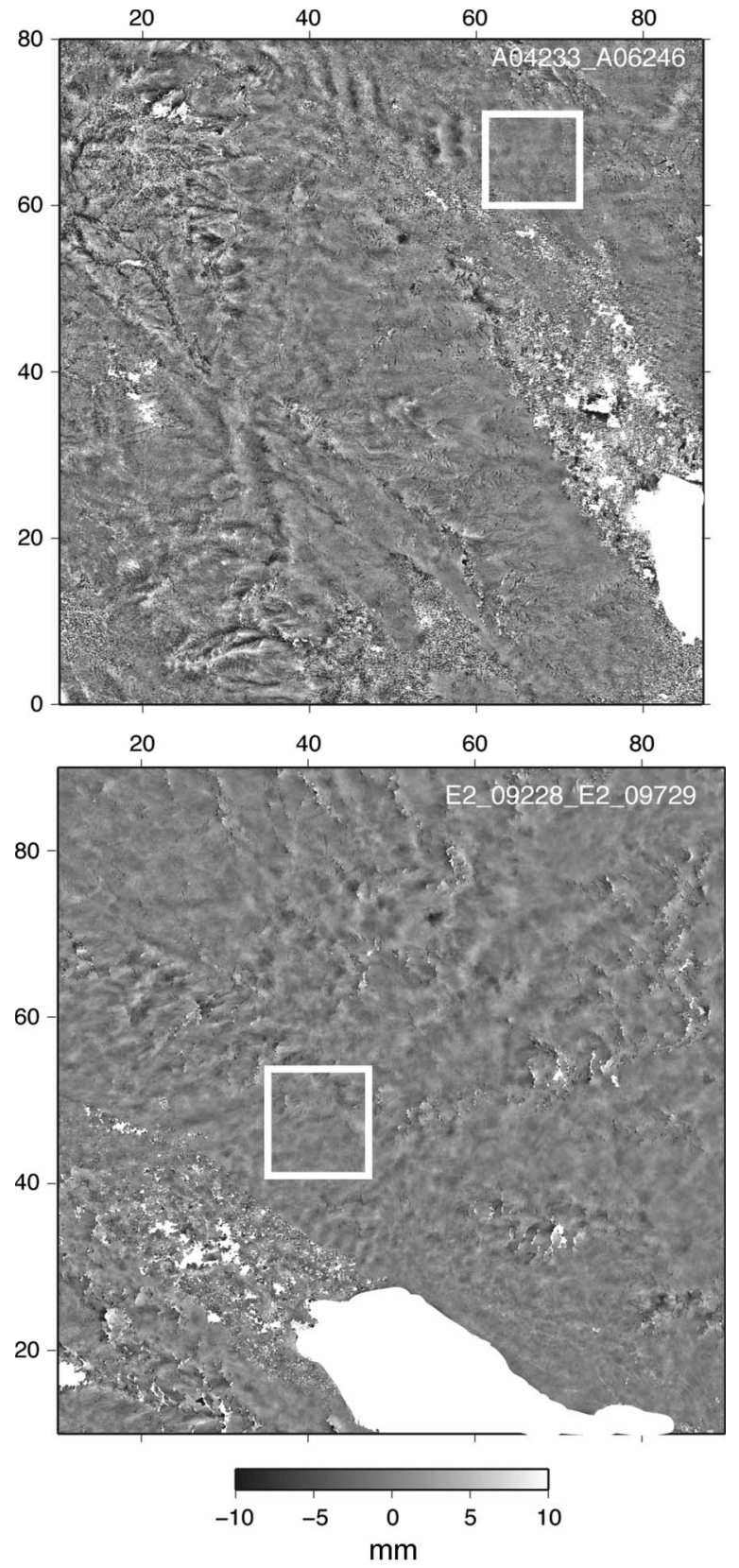

Fig. 6. LOS residual errors for (upper) ALOS and (lower) ERS after bandpass filtering between wavelengths of 100 and $5000 \mathrm{~m}$. The phase in radians was mapped to LOS by scaling by the radar wavelength divided by $4 \pi(4.5-\mathrm{mm}$ ERS and $18.8 \mathrm{~mm}$ for ALOS). Much of the residual phase error is related to topography errors, which scale directly with the baseline as well as atmospheric noise. The small subareas were selected because of low relief and good correlation.

The rms of the residual LOS for each interferogram with respect to the SRTM-topography is provided in Table III. For the full area, the average ALOS noise is $5.8 \mathrm{~mm}$ while the average ERS noise is $3.0 \mathrm{~mm}$. Part of the difference in the noise levels could be due to the longer baseline and higher topographic phase error in ALOS. Therefore, we further restricted the residual-phase contribution to a small low-relief area (white boxes in Fig. 6). In this area, the average ALOS noise is $3.3 \mathrm{~mm}$ while the average ERS noise is $2.1 \mathrm{~mm}$. This analysis suggests that the radar noise in ALOS is 1.57 greater than ERS. This is much smaller than the factor of four based on simple 
TABLE III

NOISE ALOS VERSUS ERS

\begin{tabular}{|l|c|c|c|c|c|}
\hline interferogram & mode & $\begin{array}{c}B_{\perp} \\
(\mathrm{m})\end{array}$ & $\begin{array}{c}\Delta t \\
(\text { days })\end{array}$ & $\begin{array}{c}\text { full rms } \\
(\mathrm{mm})\end{array}$ & $\begin{array}{c}\text { sub rms } \\
(\mathrm{mm})\end{array}$ \\
\hline A_4233-A_6246 & FBS2FBS & 147 & 138 & 5.80 & 2.47 \\
\hline A_6246-A_8259 & FBS2FBD & 149 & 138 & 6.68 & 3.78 \\
\hline A_7588-A_8259 & FBD2FBD & 238 & 46 & 5.11 & 3.62 \\
\hline $\begin{array}{l}\text { E2_09228- } \\
\text { E2_09729 }\end{array}$ & & 61 & 35 & 2.54 & 1.69 \\
\hline $\begin{array}{l}\text { E2_14238- } \\
\text { E2_14729 }\end{array}$ & & 100 & 35 & 3.14 & 2.38 \\
\hline $\begin{array}{l}\text { E2_15240- } \\
\text { E2_15741 }\end{array}$ & & 119 & 35 & 3.13 & 2.15 \\
\hline $\begin{array}{l}\text { E2_21386- } \\
\text { E1_23390 }\end{array}$ & & 85 & 140 & 3.02 & 2.05 \\
\hline $\begin{array}{l}\text { E1_23891- } \\
\text { E2_04218 }\end{array}$ & & 20 & 71 & 3.11 & 2.19 \\
\hline
\end{tabular}

wavelength-scaling arguments. Moreover, the other contributions to small-scale phase error from the topography error and atmosphere still dominate. This rms analysis based on only three interferograms suggests that the noise level of the FBS2FBS interferogram is $1.5 \times$ lower than the noise in the two using the lower bandwidth FBD data. This $\sqrt{2}$ change in noise level is consistent with a $2 \times$ more phase samples available at the higher bandwidth.

\section{Accuracy of Vector Deformation Maps at Kilauea Hawaii}

Between June 17 and June 20, 2007, the East Rift of Kilauea Volcano opened more than $1.9 \mathrm{~m}$ as inferred from continuous GPS measurements and measured ALOS interferometry. We have analyzed two ALOS interferograms-ascending and descending - that span the event. The ascending interferogram shown in Fig. 7(a) consists of FBD acquisitions on May 5, 2007 and June 20, 2007, having a perpendicular baseline of $326 \mathrm{~m}$. A visual comparison of this interferogram with a similar timeperiod interferogram from ENVISAT [19] shows much better phase recovery at L-band than C-band that is in agreement with a previous study of the same area using simultaneous L- and C-band acquisitions from the space shuttle [3]. The interferometric phase was unwrapped with a single seed point using the Goldstein unwrapping algorithm [14] and scaled to LOS deformation in millimeters. A second descending interferogram shown in Fig. 7(b) consists of an FBD acquisition on February 28, 2007 and an FBS interferogram on July 16, 2007 with a perpendicular baseline of $260 \mathrm{~m}$. Again, the interferometric phase is completely unwrapped with a single seed point and scaled to millimeters. In addition to the two LOS components, a third component (azimuthal offsets) can be derived from the reference and repeat images of the ascending interferogram [Fig. 7(c)]. These three components are nearly orthogonal and, thus, provide the full vector displacement for this event. The crustal deformation shows approximately $1.9 \mathrm{~m}$ of opening perpendicular to the rift zone, which is accompanied by about $0.4 \mathrm{~m}$ of rift flank uplift. Both ascending and descending interferograms show about $60 \mathrm{~mm}$ of deformation at Kilauea caldera that is consistent with $100 \mathrm{~mm}$ of subsidence. These near-field InSAR data will be used, together with the more
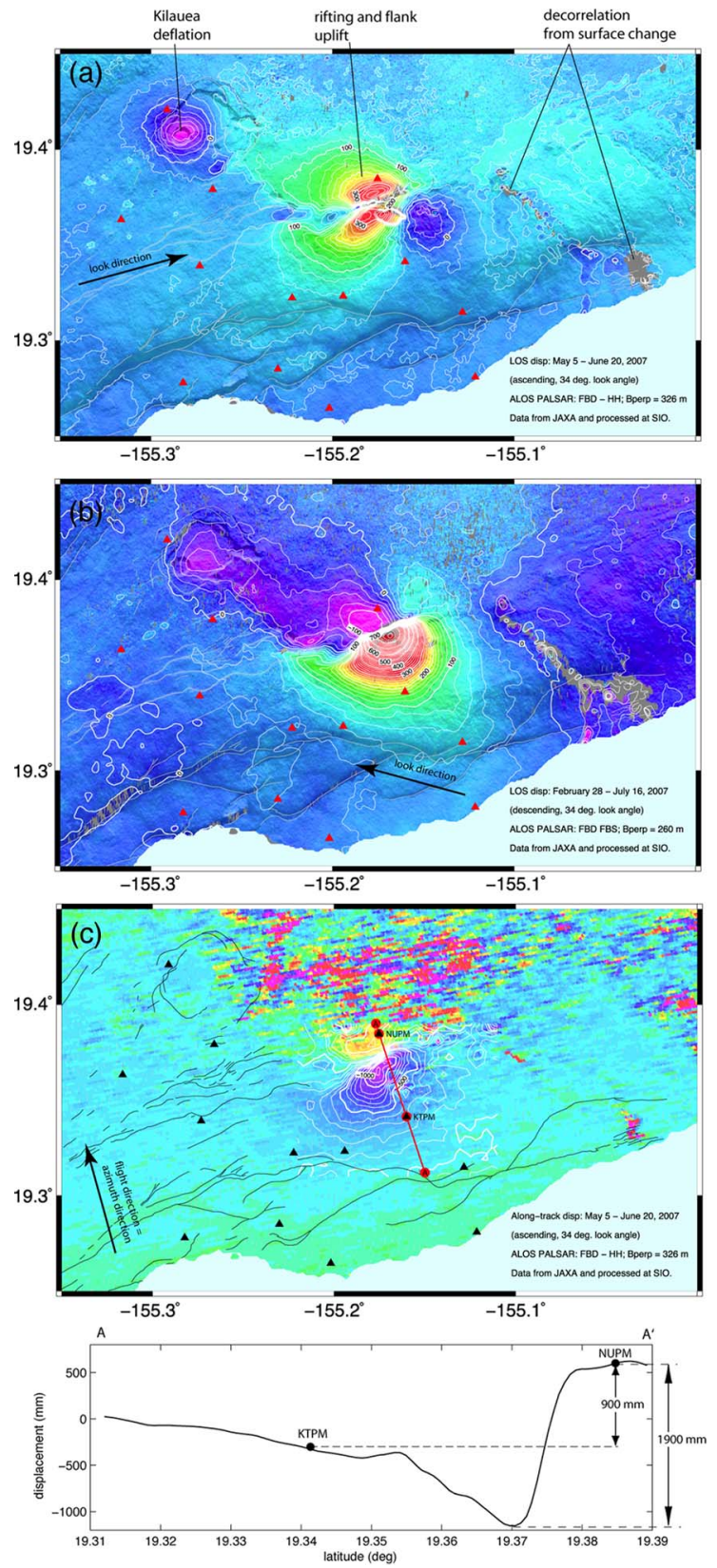

Fig. 7. (a) Radar interferogram constructed from ALOS PALSAR acquisitions on May 5 and June 20 (day 171, 8:52 GMT). This time period spans most of the "Father's day" (June 17-20) rift event. These data were acquired in the FBD polarization mode (FBD- $\mathrm{HH}, 14 \mathrm{MHz}$ ). Correlation is high even in forested areas, and the phase was unwrapped and scaled to LOS millimeters. The radar-look direction is from the WSW and $34^{\circ}$ from vertical. GPS receivers with continuous vector measurements are marked by red triangles. (b) Radar interferogram constructed from ALOS PALSAR acquisitions on Feb 28 and July 16 (8:52 GMT). This time period spans the "Father's day" rift event. These data were acquired in the two modes. The February 28 acquisition was FBD-HH (14 MHz), while the July 16 acquisition was FBS (28 MHz); the raw FDB data were interpolated to the higher FBS sampling rate. The radarlook direction is from the ESE and $34^{\circ}$ from vertical. (c) Crustal displacement in the flight direction from ALOS PALSAR acquisitions on May 5 and June 20 (day 171, 8:52 GMT). Displacements derived from cross correlation of image patches and scaled to $\mathrm{mm}$. This component of displacement is perpendicular to the LOS displacement and also about $5 \times$ less accurate. GPS receivers with continuous vector measurements are marked by black triangles. A displacement profile extracted along the line $\mathrm{A}-\mathrm{A}^{\prime}$ shows excellent agreement with the baseline change between GPS sites NUPM and KTPM. This third component reveals a peak surface separation across the rift zone of $1.86 \mathrm{~m}$, which is not fully captured $(0.90 \mathrm{~m})$ by the widely spaced GPS measurements. GPS data can be found at http://www.soest.hawaii.edu/pgf/SEQ/. 
precise far-field GPS measurements, to constrain the models of dike opening and caldera deflation associated with this event.

As a final test of the overall accuracy of the vector displacement derived from this ALOS interferometry of the Father's day rift event, we compared the LOS displacement from the ascending and descending interferograms with the displacements derived from the continuous GPS data. The GPS vector displacements were calculated from the median of the daily solutions over $+/-7$ days surrounding each SAR acquisition except for the June 20 acquisition where a $+/$-3-day window was used, because the deformation rate was high. Formal errors in the GPS vectors were typically $0.6 \mathrm{~mm}$ in the horizontal and $1.5 \mathrm{~mm}$ in the vertical, which is less than the expected errors in the interferograms. The GPS displacement vectors were projected into the two LOS components, as well as the azimuth offset component. The mean value of the interferograms was adjusted to best match the corresponding GPS LOS data. In addition, a trend was removed from the descending interferogram to flatten the displacement field far from Kilauea. No trend was removed from the ascending interferogram. The results of this comparison are shown in Fig. 8, where we have plotted LOS or azimuth displacement from the interferometry against the more accurate GPS measurements. In the comparison, 19 GPS locations were used. The two LOS components have standard deviation of 14.0 and $13.5 \mathrm{~mm}$, while the azimuth component has a $5 \times$ larger standard deviation of $70.7 \mathrm{~mm}$.

\section{CONCLUSION}

We have assessed the resolution, precision, and accuracy of ALOS interferometry using SAR images from the highbandwidth mode (28-MHz FBS), the lower bandwidth mode (14-MHz FBD), as well as mixed-mode interferograms. We find the following conditions.

1) Baseline decorrelation-The critical baseline of ALOS is $6.5 \mathrm{~km}$ for the FBD2FBD interferometry and $13 \mathrm{~km}$ for the FBS2FBS interferometry. None of our possible interferometric baselines exceeded $3 \mathrm{~km}$, and newer data have baselines less than $0.5 \mathrm{~km}$. Therefore, baseline decorrelation is not an issue with ALOS. However, errors due to inaccurate topographic phase can dominate interferometric pairs with baselines longer than about $0.2 \mathrm{~km}$. Therefore, either a shorter baseline must be used for measuring deformation or the accuracy of the global topographic grids must be improved. Moderate-baseline $(\sim 1 \mathrm{~km})$ ALOS interferograms could be used to improve the accuracy of SRTM topography.

2) Spatial resolution-Using topography as a common signal in independent interferograms, we find that the best spatial resolution (1/4 wavelength) achievable with ALOS is $38 \mathrm{~m}$ in range and $30 \mathrm{~m}$ in azimuth. This is slightly better than the resolution from Tandem ERS-1/2 interferometry ( 57 and $45 \mathrm{~m}$ ). The improvement is due to the longer critical baseline of ALOS. We do not find any significant differences in spatial resolution between the FBF2FBS, FBS2FBD, and FBD2FBD interferograms, and note that our test interferograms had baselines all less than $10 \%$ of the critical.
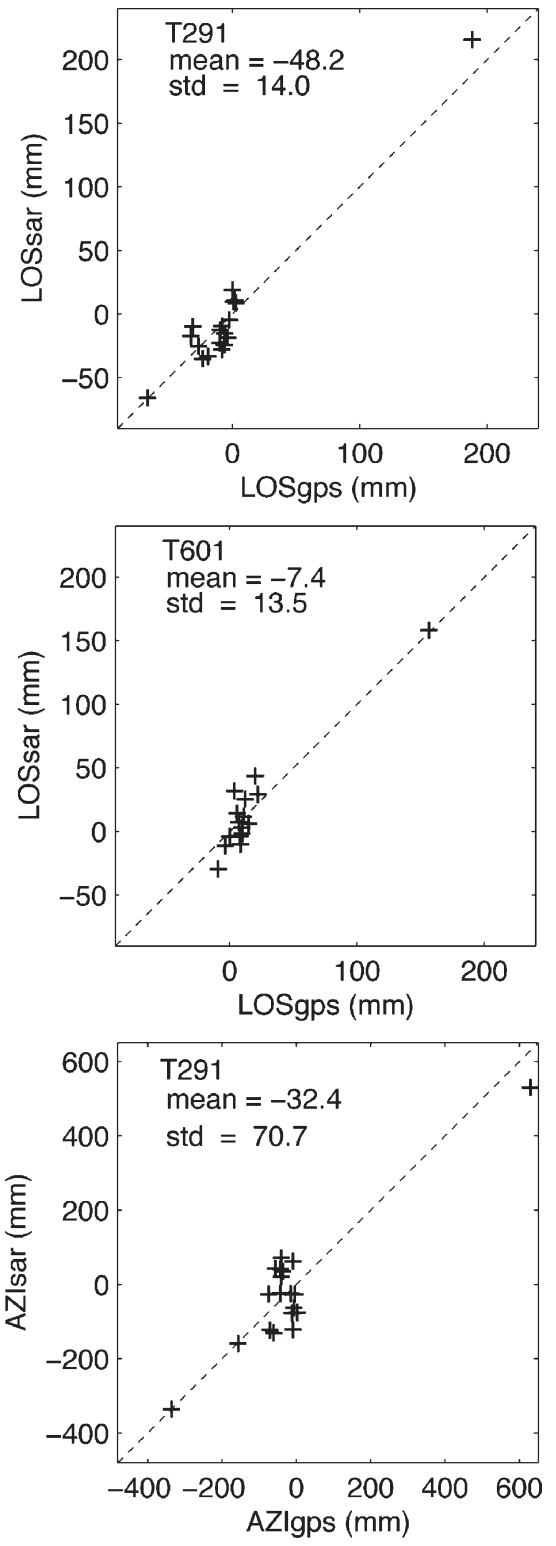

Fig. 8. Comparisons of deformation from noisy ALOS interferometry with GPS vector displacements derived from a continuous measurements surrounding Kilauea (red triangles in Fig. 7).

3) Radar noise-Simple wavelength-scaling arguments predict that the LOS range precision of L-band interferometry should be $4 \times$ worse than C-band interferometry. These arguments are incorrect in the case of ALOS PALSAR, where we find that LOS range precision of ALOS is only $1.5 \times$ worse than ERS. Again, we find that the LOS range precision is relatively independent of radar mode.

4) Overall accuracy-The June 17, 2007 rift event at Kilauea provides an optimal signal for assessing the overall accuracy of ALOS interferometry. Comparisons of 19 GPS vectors projected into the LOS of the ascending and descending interferograms show an rms deviation of $14 \mathrm{~mm}$. The surprising result is that the azimuth offsets show a standard deviation of $71 \mathrm{~mm}$. This is a remarkable result considering $71 \mathrm{~mm}$ is only $2 \%$ of the azimuth pixel size. The high precision of the azimuth offsets could be 
due to a combination of increased aperture length and low phase noise. The implication is that four components of displacements can be extracted from just two interferograms when the signal is large $(>200 \mathrm{~mm})$ and the coherence is high.

5) Atmosphere and ionosphere errors-As expected, we see phase errors that are probably due to tropospheric water vapor. Ionospheric errors should be $16 \times$ worse at L-band, and we have not assessed the longer wavelength ionosphere and orbital errors in this analysis.

\section{APPENDIX A \\ ALGORITHM FOR DOPPLER RATE}

The longer synthetic aperture of PALSAR ( 9000 echoes), with respect to ERS/ENVISAT (2800 echoes) coupled with the improved range resolution, requires a $6 \times$ more precise description of the range-migration path. In the range Doppler SAR processing algorithm, this corresponds to a more accurate of Doppler centroid and Doppler rate. Other SAR processing algorithms such as the range-migration algorithm and chirp scaling [20] also require an accurate description of the rangemigration path to achieve proper focus. Fortunately, the ALOS orbital information supplied with the raw data has sufficient accuracy to estimate these parameters. To properly account for the range variations due to the elliptical orbit and Earth rotation, we estimate the parameters by selecting a ground target somewhere in the scene and calculate the range to the target versus slow time $s$. The parabolic approximation to the range is

$$
R(s)=R_{o}+\dot{R}_{o}\left(s-s_{o}\right)+\frac{\ddot{R}_{o}}{2}\left(s-s_{o}\right)^{2}+\cdots
$$

where $R_{o}$ is the closest approach of the spacecraft to the target and $s_{O}$ is the time of closest approach. The Doppler centroid $f_{\mathrm{DC}}$ and the Doppler frequency rate $f_{\mathrm{R}}$ are related to the coefficients of this polynomial. The relationships are

$$
f_{\mathrm{DC}}=\frac{-2 \dot{R}}{\lambda} \quad f_{\mathrm{R}}=\frac{2 \ddot{R}}{\lambda}
$$

where $\lambda$ is the wavelength of the radar. In addition, if one assumes a linear trajectory of the spacecraft $V$ relative to the target, then the Doppler centroid and Doppler rate can be approximately related to the velocity and closest range [6] as

$$
f_{\mathrm{DC}}=\frac{-2 V}{\lambda} \frac{\left(x-s_{o} V\right)}{R_{o}} \quad f_{\mathrm{R}}=\frac{2 V^{2}}{\lambda R_{o}} .
$$

We will not consider the Doppler centroid further, because it is accurately estimated from the raw signal data [21]. For C-band SARs, such as ERS-1 and Envisat, the earlier formula for the $f_{\mathrm{R}}$ assuming a linear trajectory over the length of the aperture provides adequate focus. However, for L-band SARs, such as ALOS, the aperture is much longer so other factors must be considered such as the curvature and ellipticity of the orbit, as well as the rotation rate of the Earth.

Curlander and McDonough [6] discuss the estimation of the Doppler rate, and there are two main approaches. The autofocus approach uses the crudely focused imagery to improve on the estimate of the Doppler rate. The orbit approach uses the more precise geometry of the elliptical orbit about a rotating elliptical Earth to provide a more exact estimate of $\ddot{R}$. Here, we consider a new, and more direct, approach in estimating $\ddot{R}$. Consider the following three vectors, where

$\vec{R}_{s} \quad$ vector position of the satellite in the Earth-fixed coordinate system;

$\vec{R}_{e} \quad$ vector position of a point scatterer on the Earth and somewhere in the SAR scene;

$\vec{R}_{o} \quad$ LOS vector between the satellite and the point scatterer. The three vectors form a triangle such that $\vec{R}_{e}=\vec{R}_{s}+\vec{R}_{o}$. The scalar range, which is a function of slow time, is given by

$$
R(s)=\left|\vec{R}_{s}(s)-\vec{R}_{e}\right| \cong R_{o}+\dot{R}_{o}\left(s-s_{o}\right)+\frac{\ddot{R}_{o}}{2}\left(s-s_{o}\right)^{2}+\cdots
$$

Measurements of scalar range versus slow time can be used to estimate the coefficients of the parabolic approximation. The algorithm has the following functions:

1) to use the precise orbit to calculate the position vector of the satellite and compute a time series $R(s)$ over the length of the aperture;

2) to perform a least squares parabolic fit to this time series to estimate $R_{o}$ and $\ddot{R}_{o}$;

3) to compute the effective speed as $V_{e}^{2}=R_{o} \ddot{R}_{o}$ (note that it is convenient to use this effective speed in the SAR processor, because it is easily scaled with increasing range across the image). The only remaining step is to calculate the vector from the satellite to some point target in the image. This can be any point in the image, so the selection criteria are that the point lie at the proper radius of the surface of the Earth $R_{e}=\left|\vec{R}_{e}\right|$ and the $\vec{R}_{o}$ vector is perpendicular to the velocity vector of the satellite $\vec{V}$.

To evaluate the utility of this approach with PALSAR data where the squint angle is low $\left(<1^{\circ}\right)$, we compute the time evolution of the range to that point as the satellite orbits above the rotating Earth. We consider data from a descending orbit over Koga Japan where three radar reflectors have been deployed. The image is a fine-beam single polarization having a nominal look angle of $34.3^{\circ}$. A Hermite polynomial interpolation was used to calculate the $x-y-z$ position of the satellite from 28 position and velocity vectors spaced at 60 -s intervals. Thus, the entire arc is $28 \mathrm{~min}$ or a quarter on an orbit. The accuracy of the Hermite interpolator was checked by omitting a central point and performing an interpolation using six surrounding points. The accuracy of the interpolation was found to be better than $0.2 \mathrm{~mm}$, suggesting that the 60 -s interval provides an accurate representation of the orbital arc.

Next, we compute the range to the ground point as a function of time before and after the perpendicular LOS vector $\vec{R}_{o}$. We used a before/after time interval of $3 \mathrm{~s}$, which is about twice the length of the synthetic aperture for ALOS. A second-order polynomial was fit to the range versus time function, and the three coefficients provide estimates of $R_{o}, \dot{R}_{o}$, and $\ddot{R}_{o} / 2$. The ranges versus time, as well as the residual of the fit, are shown in Fig. 9. One can learn a great deal from this exercise. First, one finds that the parabolic approximation to a hyperbola used 

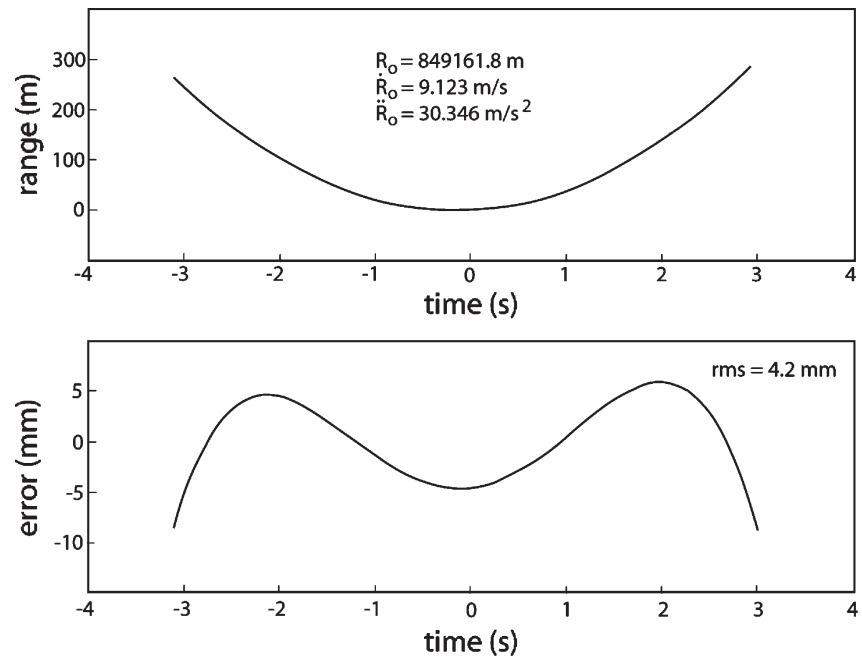

Fig. 9. (Upper) Satellite-to-ground point range as a function of time. Near range has been subtracted. The length of the synthetic aperture for ALOS PALSAR is about $\pm 1.5 \mathrm{~s}$

in the SAR processor has a maximum error of about $10 \mathrm{~mm}$ at a time offset of $3 \mathrm{~s}$. This corresponds to a small fraction of the 230-mm wavelength. Note that the actual aperture length for ALOS is only $+/-1.5 \mathrm{~s}$, so this approximation is justified as long as the squint angle is small. Second, the range at zero offset has an error of $-0.24 \mathrm{~m}$. This is due to approximating the shape of the Earth as a sphere having a local radius given by the WGS84 ellipsoid formula. In other words, there is a small error in the LOS vector because it intersects the surface of the Earth at a latitude that is slightly different from the spacecraft latitude, so the Earth radii will differ slightly. Finally, the range acceleration can be used to calculate the effective speed of the satellite $V_{e}^{2}=R_{o} \ddot{R}_{o}$. For this example, we arrive at a speed of $7174 \mathrm{~m} / \mathrm{s}$. By trial and error, we found that the optimal focus of the SAR image occurs between speeds of 7173 and $7183 \mathrm{~m} / \mathrm{s}$, which bounds our estimate. In contrast, the simple Cartesian ground-speed approximation of $7208 \mathrm{~m} / \mathrm{s}$ provided poor focus.

\section{APPENDIX B \\ ELEVATION-DEPENDENT RANGE SHIFT}

When the image baseline is long and the topographic excursions are large, the aligned resolution cells at the top of a mountain can be shifted by several range cells with respect to the aligned resolution cells at the base of the mountain. This is easily corrected by applying the known elevation-dependent range shift to the repeat single-look-complex image prior to interferogram formation. The range shift $\Delta \rho$ is given by the following:

$$
\Delta \rho=\frac{-R_{e} B_{\perp}}{\rho b \sin \theta} \Delta r
$$

where $R_{e}$ is the local Earth radius, $B_{\perp}$ is the perpendicular baseline, $\rho$ is the range, $b$ is the spacecraft orbital radius, $\theta$ is the look angle, and $\Delta r$ is the elevation change in the scene. Using nominal values for ALOS orbit and a $2.3-\mathrm{km}$ baseline of one of the Hawaii interferograms, the range shift is $-4.26 \mathrm{~m} / \mathrm{km}$ of elevation change. Mauna Loa is about $4 \mathrm{~km}$ above sea level, and the range-cell resolution of ALOS is $4.68 \mathrm{~m}$, so the pixels

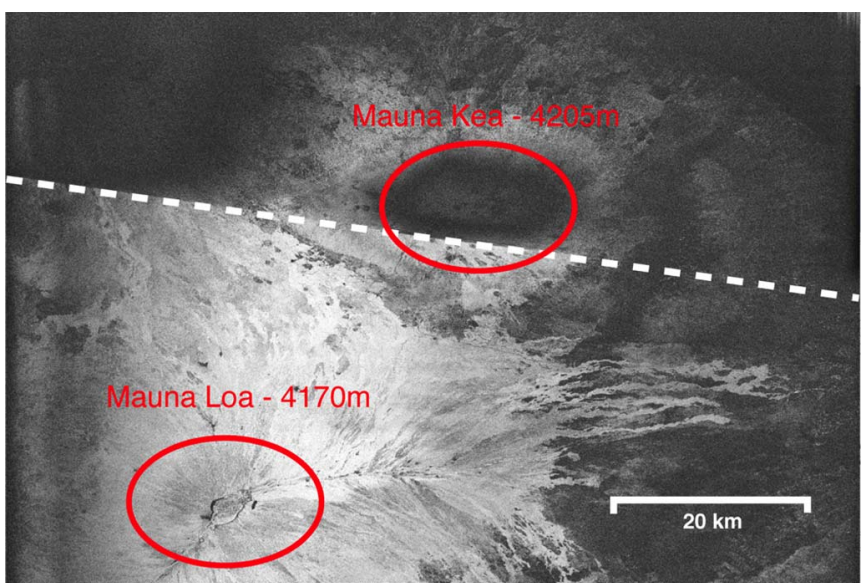

Fig. 10. Coherence of ALOS FBS2FBS interferogram over Hawaii having a perpendicular baseline of $2290 \mathrm{~m}$ (black 0.0, white 0.8). An elevationdependent range shift was applied to the lower $2 / 3$ of the interferogram (below dashed white line) and coherence is high at all elevations including the peak at Mauna Loa at $4170 \mathrm{~m}$. The area without an elevation-dependent range shift has good coherence between elevations of about 2000 and $3000 \mathrm{~m}$, but higher and lower elevations have low coherence, because pixels are misaligned in range by more than 4.7 .

are shifted by $+/-2$ resolution cells. We have verified that the correlation falls to near zero $(<0.15)$ without this correction but remains high $(\sim 0.8)$ when the correction is applied. An example is shown in Fig. 10.

\section{ACKNOWLEDGMENT}

The authors would like to thank C. Werner, P. Rosen, M. Poland, and H. Zebker for reviewing a draft version of this paper. The reviewers and associate editor provided helpful suggestions for improving this paper. GPS data from GGN and the Hawaiian GPS network were provided by SOEST and UNAVCO. Computer code to perform the mode conversion as well as to align the near range of the raw radar echoes and compute interferometric baselines is available at http://www-rohan. sdsu.edu/ mellors/ALOS_preproc.tar.gz.

\section{REFERENCES}

[1] M. Shimada, O. Isoguchi, T. Tadono, R. Higuchi, and K. Isono, "PALSAR CALVAL summary and update 2007," in Proc. IGARSS, Barcelona, Spain, 2007, pp. 3593-3596.

[2] A. Rosenqvist, M. Shimada, N. Ito, and M. Watanabe, "ALOS PALSAR: A pathfinder mission for global-scale monitoring of the environment," IEEE Trans. Geosci. Remote Sens., vol. 45, no. 11, pp. 3307-3316, Nov. 2007.

[3] P. A. Rosen, S. Hensley, H. A. Zebker, F. H. Webb, and E. Fielding, "Surface deformation and coherence measurements of Kilauea Volcano, Hawaii from SIR-C radar interferometry," J. Geophys. Res., vol. 101, no. E10, pp. 23 109-23 125, 1996.

[4] R. F. Hanssen, Radar Interferometry: Data Interpretation and Error Analysis. Boston, MA: Kluwer, 2001.

[5] D. Massonnet and K. Feigl, "Radar interferometry and its application to changes in the earth's surface," Rev. Geophys., vol. 36, no. 4, pp. 441-500, 1998.

[6] J. C. Curlander and R. N. McDonough, Synthetic Aperture Radar: Systems \& Signal Processing. New York: Wiley, 1991.

[7] G. C. Carter, C. H. Knapp, and A. H. Nuttall, "Estimation of the magnitude-squared coherence function via overlapped fast Fourier transform processing," IEEE Trans. Audio Electroacoust., vol. AU-21, no. 4, pp. 337-344, Aug. 1973.

[8] J. S. Bendat and A. G. Piersol, Random Data Analysis and Measurement Procedures, 2nd ed. New York: Wiley, 1986. 
[9] D. T. Sandwell and E. J. Price, "Phase gradient approach to stacking interferograms," J. Geophys. Res., vol. 103, no. B12, pp. 30 183-30204, Dec. 1998.

[10] H. Tarayre and D. Massonnet, "Atmospheric propagation heterogeneities revealed by ERS-1 interferometry," Geophys. Res. Lett., vol. 23, no. 9, pp. 989-992, 1996.

[11] Z. W. Xu, J. Wu, and Z. S. Wu, "A survey of ionospheric effects on space-based radar," Waves Random Media, vol. 14, no. 2, pp. S189-S273, Apr. 2004

[12] E. Chapin, S. F. Chan, B. D. Chapman, C. W. Chen, J. M. Martin, T. R. Michel, R. J. Muellerschoen, X. Pi, and P. A. Rosen, "Impact of the ionosphere on an L-band space based radar," presented at the IEEE Conf. Radar, New York, Apr. 24-27, 2006, 8 pp. DOI:10.1109/RADAR. 2006.1631775

[13] H. A. Zebker, P. A. Rosen, and R. M. Goldstein, "On the derivation of coseismic displacement fields using differential radar interferometry: The Landers earthquake," J. Geophys. Res., vol. 99, no. B10, pp. 1961719634, 1994.

[14] R. M. Goldstein, H. A. Zebker, and C. Werner, "Satellite radar interferometry: Two-dimensional phase unwrapping," Radio Sci., vol. 23, no. 4, pp. 713-720, Aug. 1988.

[15] C. Werner, S. Hensley, R. M. Goldstein, P. Rosen, and H. Zebker, "Techniques and application of SAR interferometry for ERS-1: Topographic mapping, change detection, and slope measurement," in Proc. 1st ERS-1 Symp., Cannes, France, 1992, pp. 205-210.

[16] P. D. Welch, "The use of fast Fourier transform for the estimation of power spectra: A method based on time averaging over short, modified periodograms," IEEE Trans. Audio Electroacoust., vol. AU-15, no. 2, pp. 70-73, Jun. 1967.

[17] D. T. Sandwell, L. Sichoix, D. Agnew, Y. Bock, and J.-B. Minster, "Near real-time radar interferometry of the $\mathrm{Mw} 7.1$ hector mine earthquake," Geophys. Res. Lett., vol. 27, no. 19, pp. 3101-3104, Oct. 2000.

[18] T. G. Farr, P. A. Rosen, E. Caro, R. Crippen, R. Duren, S. Hensley, M. Kobrick, M. Paller, E. Rodrigues, L. Roth, D. Seal, S. Shaffer, J. Shimada, J. Umland, M. Werner, M. Oskin, D. Burbank, and D. Alsdorf, "The shuttle radar topography mission," Rev. Geophys., vol. 45, no. 2, p. RG2 004, May 2007.

[19] M. Poland, A. Miklius, T. Orr, J. Sutton, C. Thornber, and D. Wilson, "New episodes of volcanism at Kilauea Volcano, Hawaii," EOS, Trans. Amer. Geophys. Union, vol. 89, no. 5, pp. 37-38, Jan. 2008.

[20] R. K. Raney, H. Runge, R. Balmer, I. G. Cumming, and F. H. Wong, "Precision SAR processing using chirp scaling," IEEE Trans. Geosci. Remote Sens., vol. 32, no. 4, pp. 786-799, Jul. 1994.

[21] S. N. Madsen, "Estimating the Doppler centroid of SAR data," IEEE Trans. Aerosp. Electron. Syst., vol. 25, no. 2, pp. 134-140, Mar. 1989.

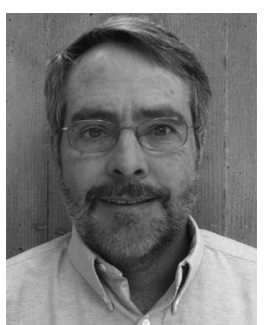

David T. Sandwell received the B.S. degree in physics from the University of Connecticut, Storrs, in 1975 and the Ph.D. degree in geophysics and space physics from the University of California at Los Angeles, Los Angeles, in 1981.

Since 1994, he has been a Professor in geophysics with the Scripps Institution of Oceanography, University of California, San Diego, La Jolla. His main areas of research are in satellite geodesy, crustal deformation, and marine geophysics.

Dr. Sandwell is a member of the Advanced Land Observing Satellite PALSAR calibration and validation team. $\mathrm{He}$ is the President-elect with the Geodesy Section, American Geophysical Union and the Chair of the Western North American InSAR Consortium.

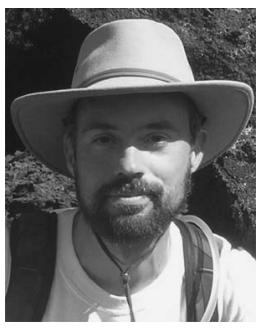

David Myer received the B.S. degree in astrophysics from the University of California, San Diego, La Jolla, in 2005, where he is currently working toward the Ph.D. degree in geophysics with a research centering on imaging volcano interiors using electromagnetic techniques in the Scripps Institution of Oceanography.

Before turning to science, he spent nearly 20 years in designing, writing, and managing commercial software projects. His interests include InSAR, GPS, and crustal-deformation modeling.

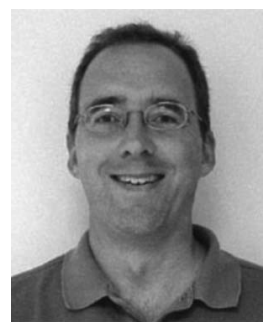

Robert Mellors received the B.S. degree in geology from The Ohio State University, Columbus, in 1986, the M.S. degree in geophysics from Cornell University, Ithaca, NY, in 1988, and the Ph.D. degree in geophysics from Indiana University, Indiana, in 1995 .

He is currently a Resident Computer Geoscientist with the Department of Geological Sciences, San Diego State University, San Diego, CA, where he teaches classes in geophysics and seismology. His research interests include earthquakes and crustal deformation (seismology and InSAR), signal processing and seismic interpretation, and earthquake education and outreach. He is a Principal Investigator on the Advanced Land Observing Satellite PALSAR team.

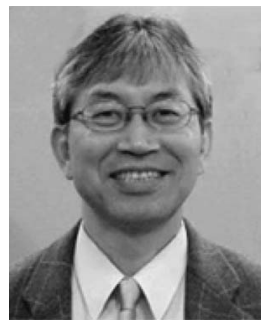

Masanobu Shimada (M'98-SM'03) received the B.S. and M.S. degrees in aeronautical engineering from Kyoto University, Kyoto, Japan, in 1977 and 1979, respectively, and the Ph.D. degree in electrical engineering from the University of Tokyo, Tokyo, Japan, in 1999.

Since 1995, he has been assigned duties with the Earth Observation Research Center, where he is in charge of the JERS-1/Advanced Land Observing Satellite (ALOS) Science Project (rainforestmapping and SAR interferometry projects). Since 1999, he has also been a Senior Scientist and an ALOS Science Manager. He is currently also with JAXA, Tsukuba Space Center, Tsukuba, Japan. His current research interests include SAR calibration and SAR interferometric applications, including polarimetric SAR interferometry (crustal-deformation detection, tree-height detection, and continental land movement).

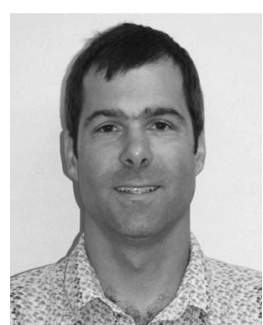

Benjamin Brooks received the B.S. degree in earth sciences from the University of California, Santa Cruz, Santa Cruz, in 1991 and the Ph.D. degree in geological sciences from Cornell University, Ithaca, NY, in 1999.

From 1999 to 2003, he was a Research Associate and Assistant Researcher with the Hawaii Institute of Geophysics, University of Hawaii, Honolulu, where he was specializing in tectonic geodesy. Since 2003, he has been the Director with the Pacific GPS Facility, University of Hawaii. His current research interests focus on using multiple geodetic platforms (GPS, InSAR, and groundbased LiDAR) to measure surface deformation and to relate the observations to tectonic, volcanic, or geomorphologic processes.

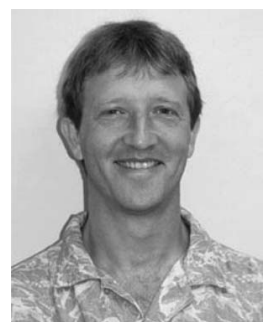

James Foster received the B.Sc. degree in geophysics from the University of Edinburgh, Edinburgh, U.K., in 1991 and the Ph.D. degree in geology and geophysics from the University of Hawaii, Honolulu, HI, in 2002.

From 2002 to 2005, he was a Research Associate with the Department of Meteorology, University of Hawaii. He is currently with the Hawaii Institute of Geophysics, University of Hawaii, where he was, first, a Research Associate and, then, an Assistant Researcher. His current research interests involve measuring and mitigating the impact of the troposphere on space geodetic measurements in support of both meteorological applications and studies of tectonic and volcanic processes. 